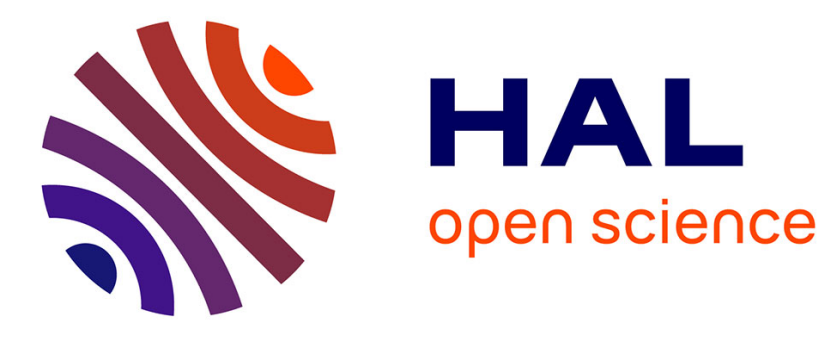

\title{
Topological Impact on Kinetic Stability of Supramolecular Polymers
}

\author{
Atsuhito Suzuki, Keisuke Aratsu, Sougata Datta, Nobutaka Shimizu, Hideaki \\ Takagi, Rie Haruki, Shin-Ichi Adachi, Martin Hollamby, Fabien Silly, Shiki \\ Yagai
}

\section{To cite this version:}

Atsuhito Suzuki, Keisuke Aratsu, Sougata Datta, Nobutaka Shimizu, Hideaki Takagi, et al.. Topological Impact on Kinetic Stability of Supramolecular Polymers. Journal of the American Chemical Society, 2019, 141 (33), pp.13196-13202. 10.1021/jacs.9b06029 . hal-02433470

\author{
HAL Id: hal-02433470 \\ https://hal.science/hal-02433470
}

Submitted on 9 Jan 2020

HAL is a multi-disciplinary open access archive for the deposit and dissemination of scientific research documents, whether they are published or not. The documents may come from teaching and research institutions in France or abroad, or from public or private research centers.
L'archive ouverte pluridisciplinaire HAL, est destinée au dépôt et à la diffusion de documents scientifiques de niveau recherche, publiés ou non, émanant des établissements d'enseignement et de recherche français ou étrangers, des laboratoires publics ou privés. 


\title{
Topological Impact on Kinetic Stability of Supramolecular Polymers
}

\author{
Atsuhito Suzuki, ${ }^{\dagger}$ Keisuke Aratsu, ${ }^{\dagger}$ Sougata Datta, ${ }^{\ddagger}$ Nobutaka Shimizu, $§$ Hideaki Takagi,, Rie Haruki, ${ }^{\S}$ Shin-ichi \\ Adachi, $\S$ Martin Hollamby, ${ }^{\perp}$ Fabien Silly, ${ }^{\complement}$ and Shiki Yagai*,+,\# \\ ${ }^{\dagger}$ Division of Advanced Science and Engineering, Graduate School of Engineering, Chiba University, 1-33 Yayoi-cho, Inage- \\ ku, Chiba 263-8522, Japan. \\ *Institute for Global Prominent Research (IGPR), Chiba University, 1-33, Yayoi-cho, Inage-ku, Chiba 263-8522, Japan. \\ \$Photon Factory, Institute of Materials Structure Science, High Energy Accelerator Research Organization, Tsukuba 305- \\ 0801, Japan. \\ ${ }^{+}$School of Physical and Geographical Sciences, Keele University, Keele, Staffordshire ST55BG, UK. \\ "TITANS, SPEC, CEA, CNRS, Université Paris-Saclay, CEA Saclay, F-91191 Gif sur Yvette, France
}

\begin{abstract}
Kinetically formed metastable molecular assemblies have attracted increasing interest especially in the field of supramolecular polymers. In most cases, metastable assemblies are ensembles of aggregates based on the same supramolecular motif but with different lengths or sizes, and therefore their kinetic stabilities are experimentally indistinguishable. Herein, we demonstrate a topological effect on kinetic stabilities in a complex mixture of metastable supramolecular polymers. Our azobenzene-incorporated monomer at ambient temperature kinetically forms complex mixtures of supramolecular polymers with cyclized and open-ended randomly coiled topologies, while upon heating we obtained thermodynamically stable crystalline fibers. Through the direct visualization of the complex supramolecular polymer mixtures by atomic force microscopy, we demonstrate that the cyclized supramolecular polymer has superior kinetic stability compared to the open-ended species toward the thermal transformation into crystalline fibers. Since the superior kinetic stability of the cyclized species can be attributed to the absence of aggregate termini, we could convert them fully into the thermodynamic species through photo-induced opening of the cyclized structures.
\end{abstract}

\section{Introduction}

Pathway complexity is becoming topical as a general concept applicable to diverse supramolecular polymer systems. ${ }^{1}$ To utilize such an intriguing concept of molecular self-assembly in order to engineer the structure and property of supramolecular polymers, and hence the resulting soft materials such as nanofibers, gels, and liquid crystalline bulk materials, one must carefully design and analyze not only thermodynamically stable products but also kinetically formed metastable species. Either through mastering intramolecular interactions ${ }^{2}$ or intermolecular interactions, ${ }^{3}$ one may rationally access metastable assemblies that enable us to kinetically control the formation of thermodynamically stable species. Although in most systems, metastable assemblies have indistinguishable kinetic stabilities despite their difference in aggregate sizes, in this study we focus on topological effect ${ }^{4}$ on kinetic stabilities in a complex mixture of metastable supramolecular polymers.

Based on the basic molecular design combining barbituric acid head group, $\pi$-conjugated core, and wedge- shaped aliphatic tails, we have explored supramolecular polymer systems of which complex landscape of welldefined higher-order structure states can be manipulated by external stimuli. ${ }^{5}$ Depending on the $\pi$-conjugated core and preparation protocols, a series of molecules form supramolecular polymers with toroidal, ${ }^{6}$ randomly coiled, ${ }^{7}$ and helically elongated topologies ${ }^{8}$ in nonpolar media. A key to these unique topological features is the hydrogen-bond-directed supermacrocyclization (primarily 6 -membered) by the barbituric acid group. The resulting supermacrocycles, so-called rosettes, give rise to the intrinsic curvature in highly idiosyncratic stacking mode with translational and rotational displacements. ${ }^{5}$ One versatile monomer is $\mathbf{1}$ (Figure 1A), whose supramolecular polymer topologies can be controlled via cooling-rate as well as light to afford random coils, rings, and helical coils (Figure 1B). ${ }^{7}$ Having this versatile monomer, we sought to understand structure-property relationships of supramolecular polymers by modifying monomer structures. To our surprise, a minor alteration of the monomer structure of $\mathbf{1}$ with the ether group linking the two 
chromophores to $\mathbf{2}$ with the ester group (Figure 1A) resulted in insoluble crystalline fibers composed of an infinite tapelike hydrogen-bonded aggregates upon cooling its hot solutions (Figure 1C). This result implies the presence of pathway complexity in the self-assembly of $\mathbf{2}$, and we should be able to obtain not only thermodynamically stable crystalline fibers but also supramolecular polymers composed of rosettes as kinetic products. This pathway complexity offers an opportunity to study the topological effect on the kinetic stability of supramolecular polymers that eventually transform into crystalline fibers by changing the hydrogen-bonding pattern.

A

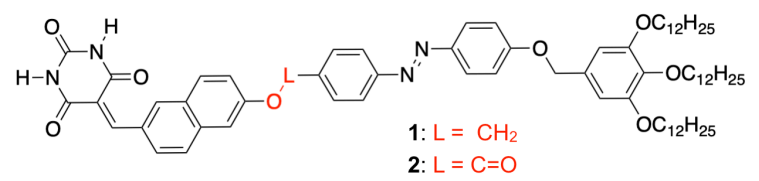

B

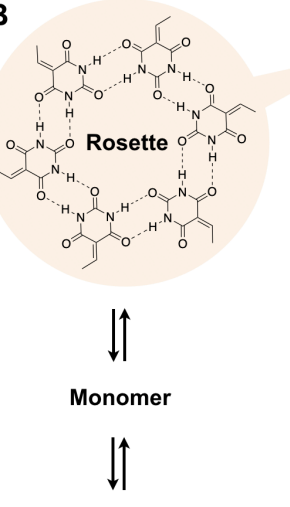

C
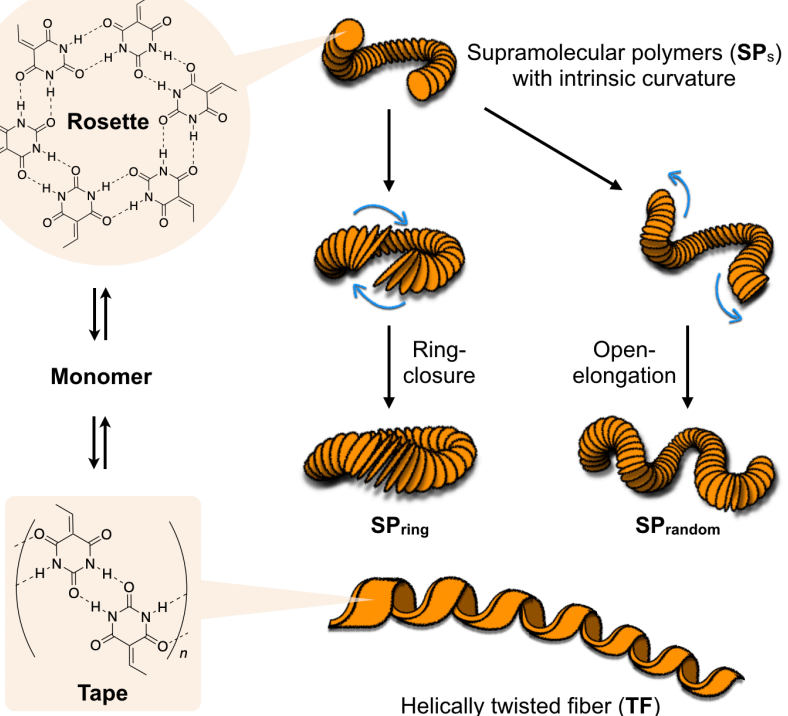

with intrinsic curvature
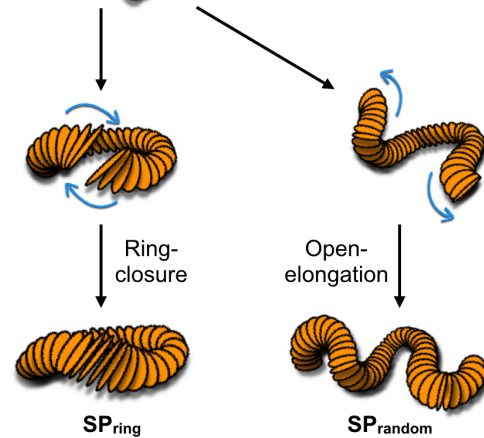

$\mathbf{S P}_{\text {ring }}$

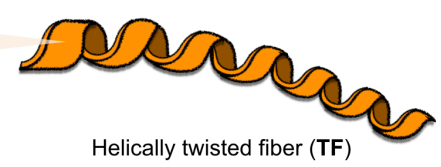

D

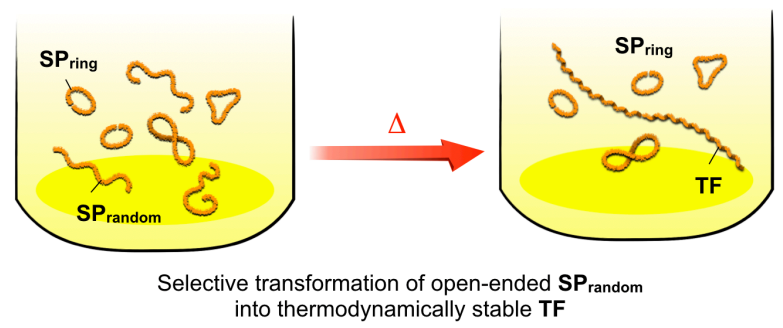

Figure 1. (A) Chemical structures of $\mathbf{1}$ and 2. (B) Schematic illustration of supramolecular polymerization of hydrogen-bonded rosette to form $\mathbf{S P}_{\text {ring }}$ and $\mathbf{S} \mathbf{P}_{\text {random. }}(\mathbf{G})$ Schematic illustration of supramolecular polymerization based on hydrogen-bonded tape to form TF. The depicted hydrogen-bonding patter has been deduced from the STM study.

Here we report pathway complexity in the selfassembly of $\mathbf{2}$ leading to two kinetically formed supramolecular polymers with closed and open-ended topologies, and one thermodynamically stable crystalline fibers. Direct visualization of complex supramolecular polymer mixtures by atomic force microscopy (AFM) led us to conclude that toroidal supramolecular polymers have superior kinetic stability compared to open-ended randomly coiled species toward the transformation into crystalline fibers triggered by thermal energy.

\section{RESULTS AND DISGUSSION}

Supramolecular Polymers: Initially we found that monomer $\mathbf{2}$ can afford two types of nanofibers with essentially different properties. One is soluble supramolecular polymers (SPs) with unique topological diversity. The SPs could be obtained upon dissolving a thin film of 2 (prepared from a chloroform stock solution) in methylcyclohexane $(\mathrm{MCH})$ by heating to $65^{\circ} \mathrm{C}$ (Figure $2 \mathrm{~A}$, top). When the resulting yellowish homogeneous solution (total concentration of monomer, $c_{\mathrm{T}}=1.0 \times$ $10^{-4} \mathrm{M}$ ) was spin-coated onto highly oriented pyrolytic graphite (HOPG) substrate, atomic force microscopy (AFM) showed a complex mixture of open-ended randomly coiled (SPP $\mathbf{S}_{\text {random }}$ ) and circular supramolecular polymer fibers $\left(\mathbf{S P}_{\text {ring }}\right.$ ) (Figure 2B, Figure S1). These structures have similar curvature radii: $r_{\text {ave }}=23.1 \pm 0.2$ $\mathrm{nm}$ for $\mathbf{S P}_{\text {random; }} 23.8 \pm 0.2 \mathrm{~nm}$ for $\mathbf{S P}_{\text {ring }}$ (Figure S2). The similarity in their intrinsic curvature implies that the internal order attained by a regular stacking of rosettes with rotational and translational displacements is identical. Accordingly, these two SPs result from competing open-ended chain elongation and ring-closure pathways of growing supramolecular polymer chains of rosettes upon dissolving 2 (Figure 1B). ${ }^{5}$ While an unidirectional stacking of rosettes about $150 \mathrm{~nm}\left(2 \pi r_{\text {ave }}\right)$ could lead to $\mathbf{S P}_{\text {ring, }}$, changes in the turning direction of the curvature en route to $\mathbf{S P}_{\text {ring }}$, caused by some defects in the unidirectional stacking of rosettes, would lead to $\mathbf{S P}_{\text {random. }}$ Reflecting the open-ended structure, the lengths of $\mathbf{S P}_{\text {random }}$ are largely distributed from 50 to $600 \mathrm{~nm}$ in comparison with those of $\mathbf{S P}_{\text {ring }}$ (Figure 2C). We could not effectively increase the frequency of the ring-closure process by changing the heating rate. The average yield of $\mathbf{S P}_{\text {ring }}$ based on the monomer was $8.4 \%$ as estimated by AFM (Figure S3).

Selective Transformation: The other type of nanofibers was obtained upon further heating the above mixture of SPs. Dynamic light scattering (DLS) of the mixture at $65 \mathrm{C}$ showed the presence of largely polydispersed assemblies in the range of $80-400 \mathrm{~nm}$, suggesting that $\mathbf{S P}_{\text {ring }}$ and $\mathbf{S P}_{\text {random }}$ are indistinguishable (Figure S4). Upon heating, the yellow color of the solution intensified around $85-90{ }^{\circ} \mathrm{C}$ (Figure 2A), and the broad DLS peak spitted into two peaks centered at $90 \mathrm{~nm}$ and $700 \mathrm{~nm}$, respectively, suggesting the formation of assemblies with distinguishable size difference (Figure S4). On prolonged heating $(3 \mathrm{~h})$ at the same temperature, precipitation occurred (Figure 2A). Optical microscopic observation of the precipitates revealed bundled fiberslike morphology exhibiting birefringence (Figure 2D). AFM observation showed that the fibers are further 

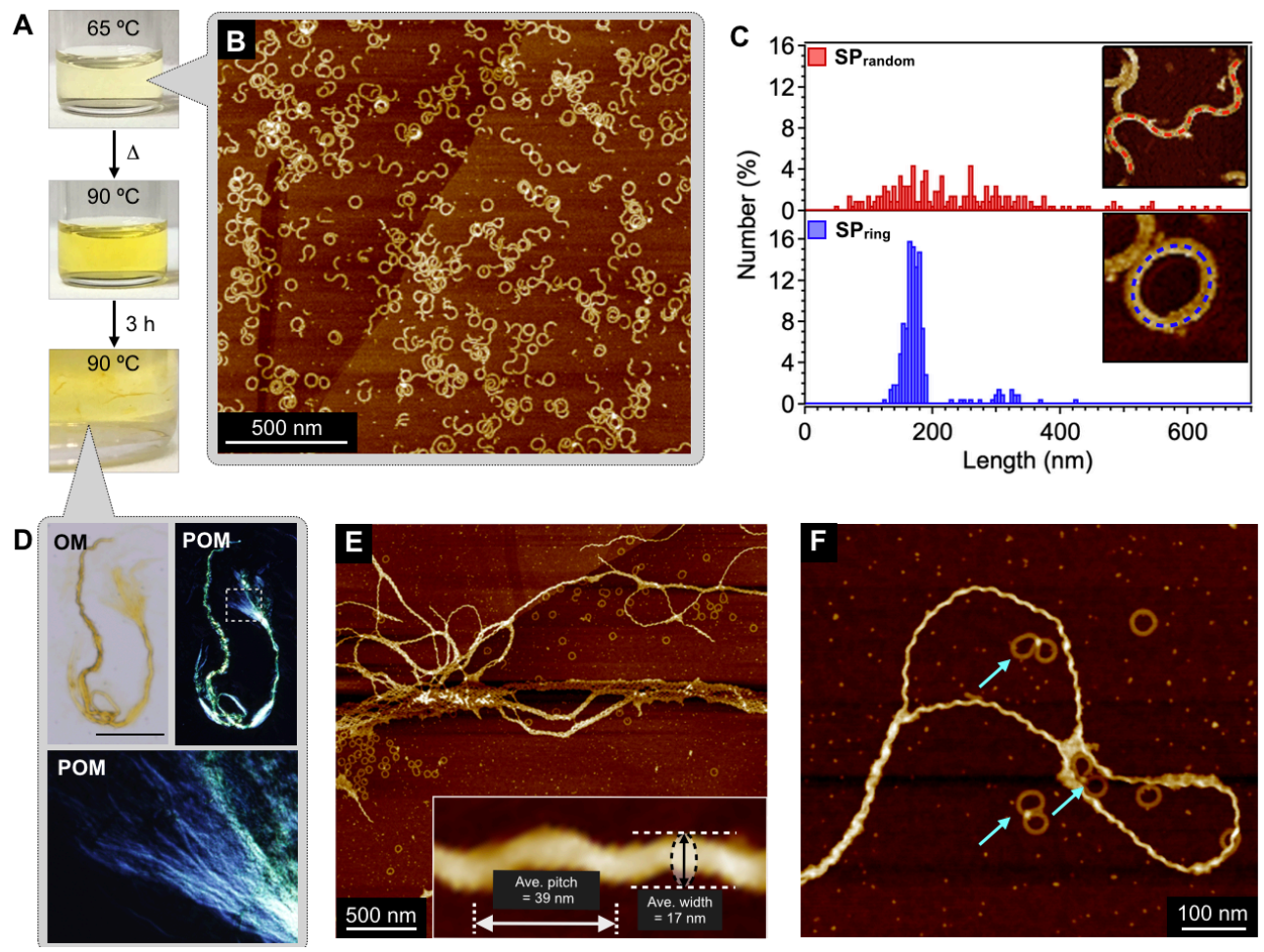

Figure 2. (A) Photographs of a MCH solution of $2\left(c_{\mathrm{T}}=1.0 \times 10^{-4} \mathrm{M}\right)$ upon heating. (B) AFM image of the structural transition of the supramolecular polymers, which were prepared by heating a $\mathrm{MCH}$ solution of $\mathbf{2}$ at a heating rate of $0.3^{\circ} \mathrm{C} \min ^{-1}$. (C) Proposed mechanism for forming cyclic structure and randomly coiled. (D) Optical micrograph and polarized optical micrographs of precipitated TF. (E,F) AFM images of $\mathbf{T F}$ and $\mathbf{S P}_{\text {ring. }}$ The blue arrows in $(\mathrm{F})$ indicate "figure eight" $\mathbf{S P}_{\text {ring. }}$.

composed of helically twisted fibrils $(\mathbf{T F})$ with average edge-to-edge width of $17 \mathrm{~nm}$ and helical pitch of $39 \mathrm{~nm}$ (Figure 2E). Interestingly, AFM imaging of the supernatant showed only $\mathbf{S P}_{\text {ring }}$ and $\mathbf{T F}$, and $\mathbf{S P}_{\text {random }}$ that existed up to $65{ }^{\circ} \mathrm{C}$ was hardly observed (Figure 2E,F). These observations indicate that $\mathbf{S P}_{\text {random }}$ can be selectively converted to $\mathbf{T F}$ upon heating, and $\mathbf{S} \mathbf{P}_{\text {ring }}$ is significantly stable at higher temperature by virtue of its closed topology.

The transformation of $\mathbf{S P}_{\text {random }}$ to $\mathbf{T F}$ was also investigated by small-angle X-ray scattering (SAXS) analysis. We previously demonstrated that the SAXS profiles of toroidal ${ }^{6 \mathrm{~b}}$ and randomly coiled $\mathrm{SPs}^{7}$ are characterized by nonperiodic oscillatory features at $Q$ range of $0.3-1.2$ $\mathrm{nm}^{-1}$, which correspond to diameter of the intrinsic curvature. Here, the mixture of SPs displayed a SAXS profile with defined scattering peaks in $Q$ range of $0.18-0.8$ $\mathrm{nm}^{-1}$ (Figure 3A, blue curve), which is very similar to the profile of randomly coiled SPs of previously reported $\mathbf{1}$. Upon heating the solution, the scattering pattern dramatically changed (Figure 3A, red curve), pointing to a significant change in the solution nanostructure, in line with a transition of $\mathbf{S P}_{\text {random }}$ to $\mathbf{T F}$. The positions of the oscillations in SAXS data arising from TF (see Supporting Information) were well-replicated by a hollow cylinder model with an outer diameter of $25 \mathrm{~nm}$ (Figure S5). This is somewhat longer than the dimension observed on the AFM images (17 nm, Figure 2), with the discrepancy most likely being attributed to a "stretch- ing" of TF along to the long axis upon adsorption to HOPG. Interestingly, we do not normally see such large structural changes on adsorption for either SPs or toroidal structures as described above, which points to $\mathbf{T F}$ as having a different structure and self-assembly pathway. The intrinsic curvature of the SPs and toroids results in a reduced contact area with the alkyl chains (lower interaction strength with HOPG) on the outside. In the case of $\mathbf{T F}$ however, the flatter tape-like structure (Figure 1C) has both a larger contact area and allows the entire molecule of $\mathbf{2}$ to contact with the HOPG surface.

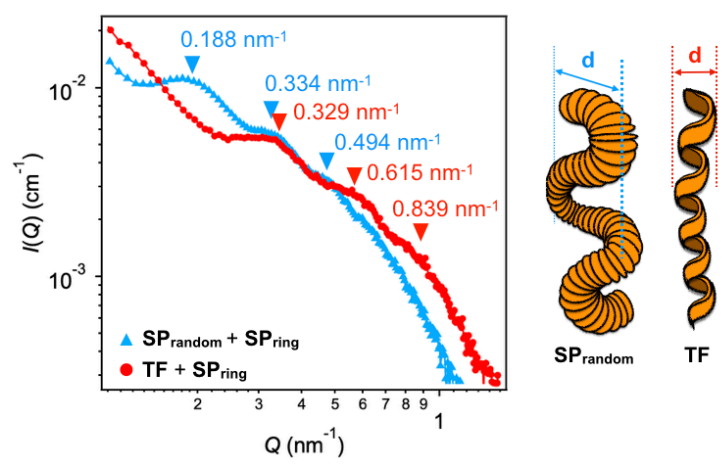

Figure 3. (A) SAXS profiles of the $\mathbf{S P}_{\text {random }}$ and $\mathbf{S P}_{\text {ring }}$ solution

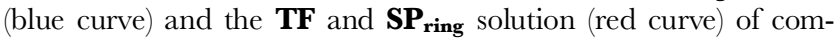
pound $2\left(c \mathrm{~T}=1.0 \times 10^{-4} \mathrm{M}\right)$. (B) Schematic representation of proposed topologies with different diameter $(d)$ arising in SAXS profiles. 
The above thermal transition was studied by temperature-dependent absorption measurements. In the temperature range $25-65{ }^{\circ} \mathrm{C}$, the mixture of SPs $\left(c_{\mathrm{T}}=\right.$ $\left.1.0 \times 10^{-4} \mathrm{M}\right)$ showed a broad absorption band at 348 $\mathrm{nm}$ corresponding to electronic transitions of barbiturated naphthalene and azobenzene chromophores of $\mathbf{2}$ (Figure 4A). This maximum is blue-shifted compared to the monomeric $2(365 \mathrm{~nm})$, attained by heating a dilute solution $\left(c_{\mathrm{T}}=1.0 \times 10^{-5} \mathrm{M}\right)$ to $90{ }^{\circ} \mathrm{C}$ (Figure $\left.4 \mathrm{~A}\right)$. The blue shift suggests a face-to-face (H-type) stacking of the naphthalene chromophore in reference to a related compound lacking azobenzene chromophore (Figure S6). Upon heating the mixture, the absorption band abruptly changed above $80^{\circ} \mathrm{C}$, and equilibrated at $85^{\circ} \mathrm{C}$ to show the maximum at $368 \mathrm{~nm}$ with the appearance of a new absorption shoulder at $460 \mathrm{~nm}$ (red curve, Figure 4B). The resulting spectrum is clearly different from that of the monomeric 2, suggesting the transformation of $\mathbf{S P}_{\text {random }}$ to thermodynamically stable aggregates $\mathbf{T F}$ comprising a slipped ( $\mathcal{f}$-type) arrangement of azobenzene stacks. The transformation was irreversible process as no absorption spectral change was observed upon subsequent cooling. The second heating analysis performed at $c_{\mathrm{T}}=3.0,2.0$ and $1.0 \times 10^{-5} \mathrm{M}$ all provided nonsigmoidal dissociation curves of $\mathbf{T F}$ into monomeric state as a function of temperature, which is characteristic of the cooperative nucleation-elongation mechanism. ${ }^{9}$ The elongation enthalpy $\left(\Delta H_{\mathrm{e}}\right)$ of $97.1 \mathrm{~kJ} \mathrm{~mol}^{-1}$ was obtained by curve fitting to the cooperative model developed by Meijer and co-workers.9a The modified van't Hoff plot ${ }^{\text {Ref }}$ using the critical elongation temperatures $\left(T_{\mathrm{e}}\right)$ estimated by the curve fitting and the monomer concentrations provided the standard enthalpy $\left(\Delta H^{\circ}\right)$ of $-113 \mathrm{~kJ} \mathrm{~mol}^{-1}$, the standard entropy $\left(\Delta S^{\circ}\right)$ of $-223 \mathrm{~J}$ $\mathrm{mol}^{-1} \mathrm{~K}^{-1}$, and the Gibbs free energy $\left(\Delta G^{\circ}\right)$ of $-48 \mathrm{~kJ}$ $\mathrm{mol}^{-1}$ (at $293 \mathrm{~K}$ ), respectively (Figure S7). The $\Delta H^{\circ}$ value is close to $\Delta H_{\mathrm{e}}$, demonstrating that TF is the thermodynamic product. ${ }^{10}$

The above spectral change occurred in relatively lower temperature range upon decreasing $c_{\mathrm{T}}$ (Figure 4B), suggesting that the dissociation of SPs to the monomeric state initiates the transformation of $\mathbf{S P}_{\text {random }}$ to $\mathbf{T F}$. To shed more light into this point, we performed timedependent absorption analysis under isothermal conditions. For this purpose, we prepared $\mathrm{MCH}$ solutions of SPs at $c_{\mathrm{T}}=2.0,2.5$ and $3.0 \times 10^{-4} \mathrm{M}$, and kept them at $65{ }^{\circ} \mathrm{C}$. For the solution with $c_{\mathrm{T}}=3.0 \times 10^{-4} \mathrm{M}$, the spectral change started after a significantly long lag time of ca. $1500 \mathrm{~min}(25 \mathrm{~h})$. The lag time became shorter upon decreasing $c_{\mathrm{T}}$ to destabilize SPs (Figure 4C), suggesting that $\mathbf{T F}$ is reorganized from monomers. la,3a Namely, SP $\mathbf{P}_{\text {random }}$ is an "off-pathway" intermediate that can be converted to $\mathbf{T F}$ via the monomeric state.
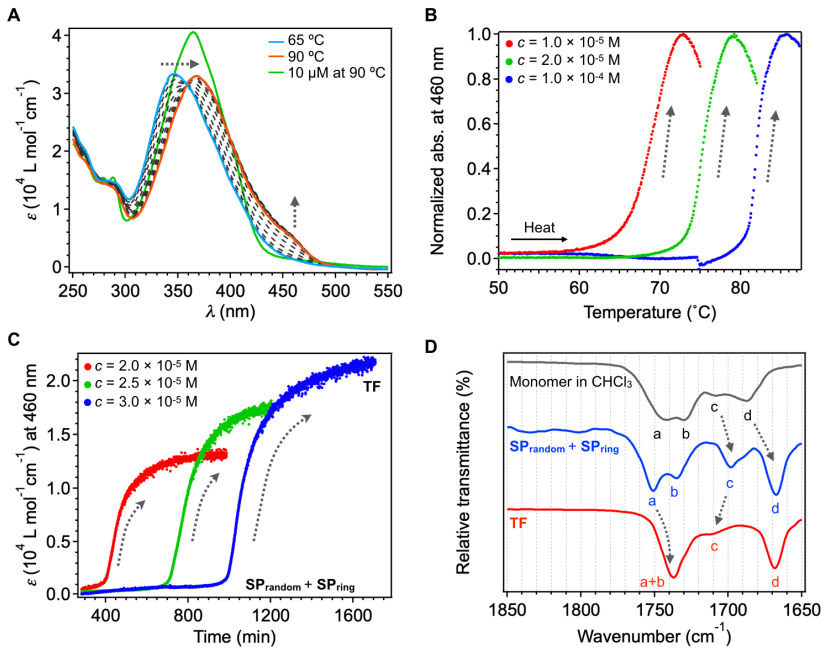

Figure 4. (A) Temperature-dependent UV-Vis spectra of $\mathrm{MCH}$ solution of $\mathbf{2}\left(c_{\mathrm{T}}=1.0 \times 10^{-4} \mathrm{M}\right)$ upon heating from 65 to $90{ }^{\circ} \mathrm{C}$ at a rate of $0.3{ }^{\circ} \mathrm{C} \mathrm{min}^{-1}$. The red arrows indicate changes upon heating. (B) Plots of normalized absorbance at $460 \mathrm{~nm}$ versus temperature upon heating $\mathrm{MCH}$ solution of 2 at $c_{\mathrm{T}}=1.0 \times 10^{-4}$ $\mathrm{M}$ (blue dots), $2.0 \times 10^{-5} \mathrm{M}$ (green dots) and $2.0 \times 10^{-5} \mathrm{M}$ (red dots). (C) Time-dependent change of $\varepsilon$ at $460 \mathrm{~nm}$ upon heating $\mathrm{MCH}$ solution of $\mathbf{2}$ at $c_{\mathrm{T}}=3.0 \times 10^{-5} \mathrm{M}$ (blue dots); $2.5 \times 10^{-5} \mathrm{M}$ (green dots); $2.0 \times 10^{-5} \mathrm{M}$ (red dots). (D) FT-IR spectrum of the solutions of compound $\mathbf{2}$ in chloroform (black line, monomer) and $\mathrm{MCH}$ (blue line, $\mathbf{S P}_{\text {random }}+\mathbf{S P}_{\text {ring }}$; red line, $\mathbf{T F}+\mathbf{S P}_{\text {ring }}$ ). $c_{\mathrm{T}}=$ $1.0 \times 10^{-4} \mathrm{M}$.

FT-IR analysis further corroborated the above results. Figure $4 \mathrm{D}$ shows the $\mathrm{C}=\mathrm{O}$ stretching vibrations of the mixture of $\mathbf{S P}_{\text {ring }}$ and $\mathbf{S P}_{\text {random, }}, \mathbf{T F}$ and monomeric $\mathbf{2}$ in chloroform. For all the spectra, four peaks can be assigned: three from barbituric acid unit and one from the ester linker. In the spectrum of the mixture, two peaks denoted $c$ and $d$ appeared at lower wavenumbers in comparison with those of the monomer. These shifts are ascribable to the hydrogen-bonding of two of three $\mathrm{C}=\mathrm{O}$ groups of the barbituric acid unit to form rosettes. Another two peaks denoted $a$ and $b$, assignable to either the remaining free $\mathrm{C}=\mathrm{O}$ or the ester $\mathrm{C}=\mathrm{O}$ group, slightly shifted higher wavenumber in the mixture because of the absence of weak hydrogen bonds with $\mathrm{CHCl}_{3}$. The spectrum of $\mathbf{T F}$ is partially different from that of the mixture. For example, peaks $a$ and $c$ attributed to the barbituric acid $\mathrm{C}=\mathrm{O}$ group are shifted to lower and higher wavenumber, respectively. The distinctly different $\mathrm{C}=\mathrm{O}$ vibration in the mixture of $\mathbf{S P}_{\text {ring }}$ and $\mathbf{S P}_{\text {random }}$ and $\mathbf{T F}$ demonstrates the rearrangement of hydrogenbonding pattern, and unequivocally demonstrates the transformation of $\mathbf{S P}_{\text {random }}$ to $\mathbf{T F}$ via the monomeric state. Although we could not characterize the exact supramolecular structure of $\mathbf{T F}$, it can be postulated that it is based on tapelike open-ended hydrogen bond motif of barbituric acid units (Figure 1C). ${ }^{11}$ In fact, scanning tunneling microscopy (STM) of $\mathbf{2}$ at a liquid-solid interface revealed a lamellar type molecular packing (Figure S8), which supports the plausibility of such a tapelike hydrogen-bonded structure. ${ }^{12}$ 


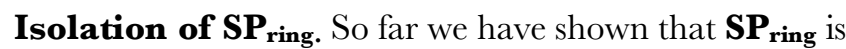
thermally stable over $\mathbf{S P}_{\text {random }}$ even under entropically favored conditions, i.e., at high temperature. To further highlight the thermal stability of $\mathbf{S P}_{\text {ring, we attempted to }}$ isolate them by passing the heated solution $\left(c_{\mathrm{T}}=1.7 \times\right.$ $10^{-4} \mathrm{M}$ ) through a membrane filter with pore size of 200 $\mathrm{nm}$ to remove $\mathbf{T F}$ (Figure 5A,B). AFM measurements demonstrated the presence of $\mathbf{S P}_{\text {ring }}$ in the filtrate predominantly, while $\mathbf{T F}$ was totally filtrated out (Figure $5 \mathrm{C}$, D). From the absorbance of the filtrate, the concentration of $\mathbf{2}$ that formed $\mathbf{S} \mathbf{P}_{\text {ring }}$ was estimated to be $1.2 \times$ $10^{-5} \mathrm{M}\left(7.2 \mathrm{~mol} \%\right.$ of $\left(c_{\mathrm{T}}\right)$, which is in good agreement with the direct estimation using AFM images $(8.4 \mathrm{~mol} \%$, Figure S3). By evaporating this filtrate, we obtained a

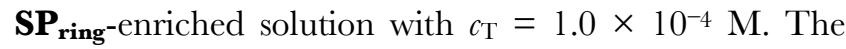
SAXS measurement of this $\mathbf{S P}_{\text {ring-enriched solution }}$ displayed more exquisite oscillations attributed to the intrinsic curvature in comparison with the mixture with SP random (Figure 5E). The SAXS data was analyzed using a model representing a delta distribution of toroids with ellipsoidal core-shell cross-sections (Figure S9), ${ }^{6 \mathrm{~b}}$ yielding a curvature radius of $21 \mathrm{~nm}$ and other structural parameters as described in Supporting Information that are in line with AFM results and the structure of $\mathbf{2}$. The absorption spectrum of this $\mathbf{S P}_{\text {ring-enriched solution did }}$ not show significant change upon heating above $80^{\circ} \mathrm{C}$, especially an increase of absorption at $460 \mathrm{~nm}$ as an indication of the transformation to $\mathbf{T F}$ (Figure 5F). After cooling, an almost comparable amount of $\mathbf{S P}_{\text {ring }}$ was observed by AFM, suggesting that $\mathbf{S P}_{\text {ring }}$ are indeed thermally stable species.

Our results demonstrated that $\mathbf{S P}_{\text {ring }}$ are either thermodynamically or kinetically inert against dissociation to monomeric state. In an interesting observation on dynamic monomer exchange pathway in a SP system using super-resolution spectroscopy, Meijer et al. has demonstrated that monomer exchange can occur homogeneously from SP chains most likely due to defects. ${ }^{13}$ Pavan et al., based on their atomistic and coarse-grained modelling studies, suggested that monomer exchange can originate from defects present in the SP chains. ${ }^{14}$ In terms of defects in our system, $\mathbf{S P}_{\text {ring, }}$, wherein rosettes stack with fully unidirectional rotations, can be regarded as a perfect structure without defect. However, it is unlikely that this enthalpic gain of $\mathbf{S P}_{\text {ring }}$ can compensate its entropic cost associated with ring-closure, and imparts thermodynamic stability superior to that of $\mathbf{S P}_{\mathbf{r a n}}$ dom. Indeed, not only $\mathbf{S P}_{\text {ring, but also much larger circu- }}$ lar species with obvious defects were found as "figure eight" shaped structures after heating (blue arrows in Figure 2G,5D). In this context, $\mathbf{S P}_{\text {ring }}$ are only kinetically stable against dissociation, probably due to the absence of reactive "termini". 15
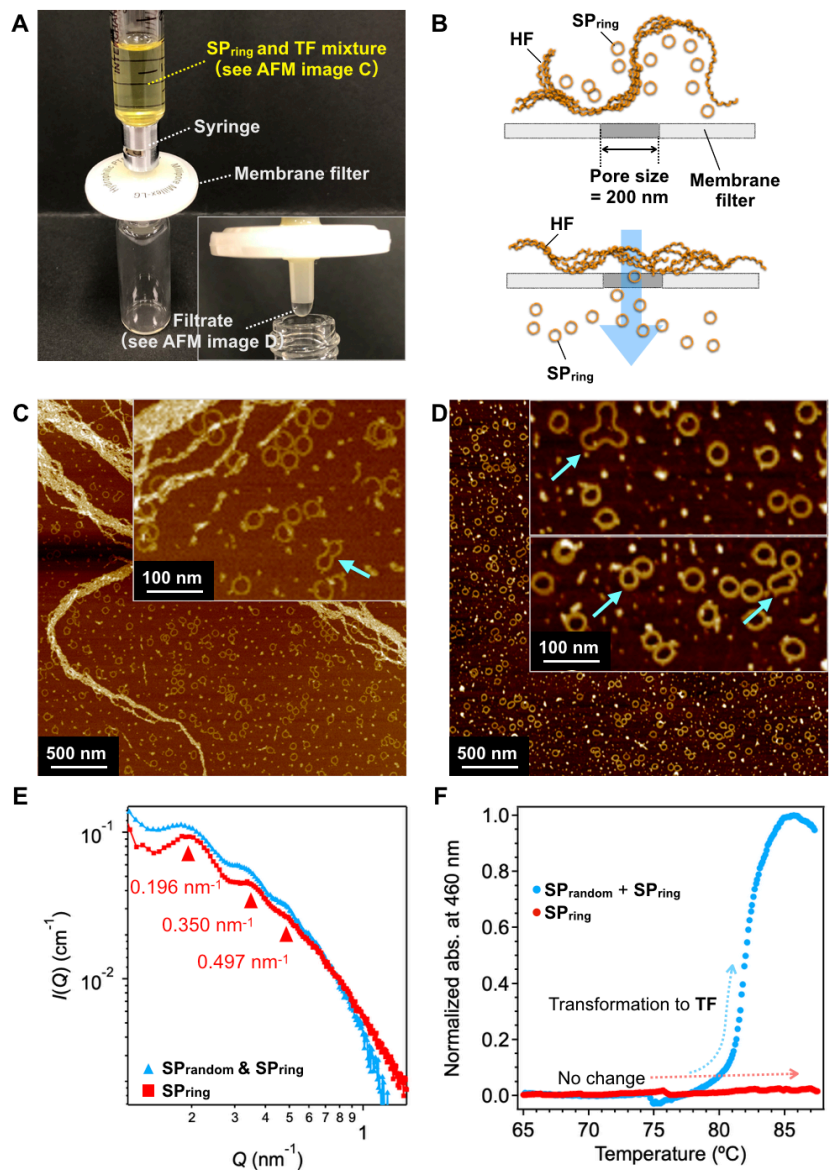

Figure. 5. (A) Photographs of filtration experiment of the mixture of $\mathbf{T F}$ and $\mathbf{S P}_{\text {ring }}$ using membrane filter. (B) Schematic illustration of selective passage of $\mathbf{S P}_{\text {ring }}$ through a pore of the membrane filter. (C, D) AFM images taken before $(\mathrm{C})$ and after $(\mathrm{D})$ filtration. (E) The SAXS profile of the filtrate (red dots) after evaporation to adjust $c_{\mathrm{T}}$ to $1.0 \times 10^{-4} \mathrm{M}$. The SAXS profile of the mixture of $\mathbf{S P}_{\text {random }}$ and $\mathbf{S} \mathbf{P}_{\text {ring }}$ is also shown for comparison (blue dots). (F) Heating curves of $\mathbf{S P}_{\text {ring }}$ (red dots) and the mixture of $\mathbf{S P}_{\text {random }}$ and $\mathbf{S P}_{\text {ring }}$ (blue dots) in $\mathrm{MCH}\left(c_{\mathrm{T}}=1.0 \times 10^{-4} \mathrm{M}\right)$ obtained by plotting absorbance at $460 \mathrm{~nm}$ as a function of temperature.

Photoinduced Ring-opening. To further shed light into the topological effect on the kinetic stability of $\mathbf{S P}_{\text {ring, we }}$ attempted to convert $\mathbf{S} \mathbf{P}_{\text {ring }}$ into open-ended short SPs by means of the trans-to-cis photoisomerization of the azobenzene moiety. Our previous study on SPs of $\mathbf{1}$ demonstrated that UV-irradiation induces a considerable conformational change of the SPs as a result of the loss of the intrinsic curvature caused by the trans-to-cis isomerization of the azobenzene moieties. ${ }^{7,16} \mathrm{UV}-\mathrm{Vis}$ and NMR spectroscopic analyses showed that the irradiation of the mixture of $\mathbf{S P}_{\text {ring }}$ and $\mathbf{S P}_{\text {random with UV }}$ light $(\lambda=365 \mathrm{~nm})$ converted $17 \mathrm{~mol} \%$ of trans- 2 into cis2 (Figure 6A, S10). In SAXS analysis, the UV-irradiated solution exhibited a featureless profile with no oscillations, in line with a loss of intrinsic curvature (Figure 6B). In AFM imaging, SP $\mathbf{P}_{\text {ring }}$ was hardly found, and only open-ended short fibers lacking the intrinsic curvature were visualized (Figure 6D). Although the majority of 
these open-ended fibers are thought to be photodeformed products of $\mathbf{S P}_{\text {random, a closer inspection of }}$ the AFM images revealed the presence of short fibers with lengths of approximately $100-300 \mathrm{~nm}$, which is consistent with the circumference (ca. $145 \mathrm{~nm}$ ) of $\mathbf{S P}_{\text {ring }}$ (pink arrows in inset of Figure 5D). Upon heating the UV-irradiated solution to $90^{\circ} \mathrm{C}$, DLS showed an entire shift of aggregate sizes above $1 \mu \mathrm{m}$, which is very contrasting to the result without UV-irradiation (Figure S4), wherein $\mathbf{S P}_{\text {ring }}$ could be detected even after heating. The absence of $\mathbf{S P}_{\text {ring }}$ was also confirmed by AFM, showing only heavily bundled $\mathbf{T F}$ without any trace of $\mathbf{S P}_{\text {ring }}$ (Figure 6E).

A

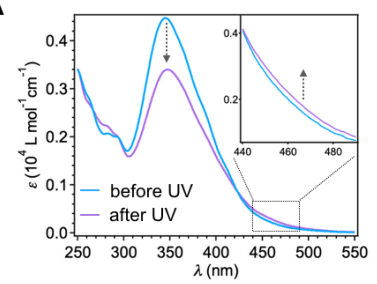

B

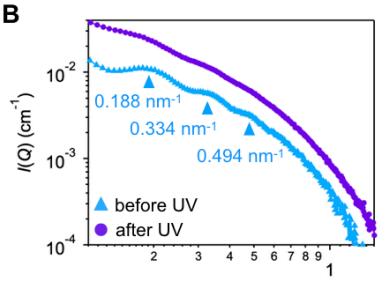

C
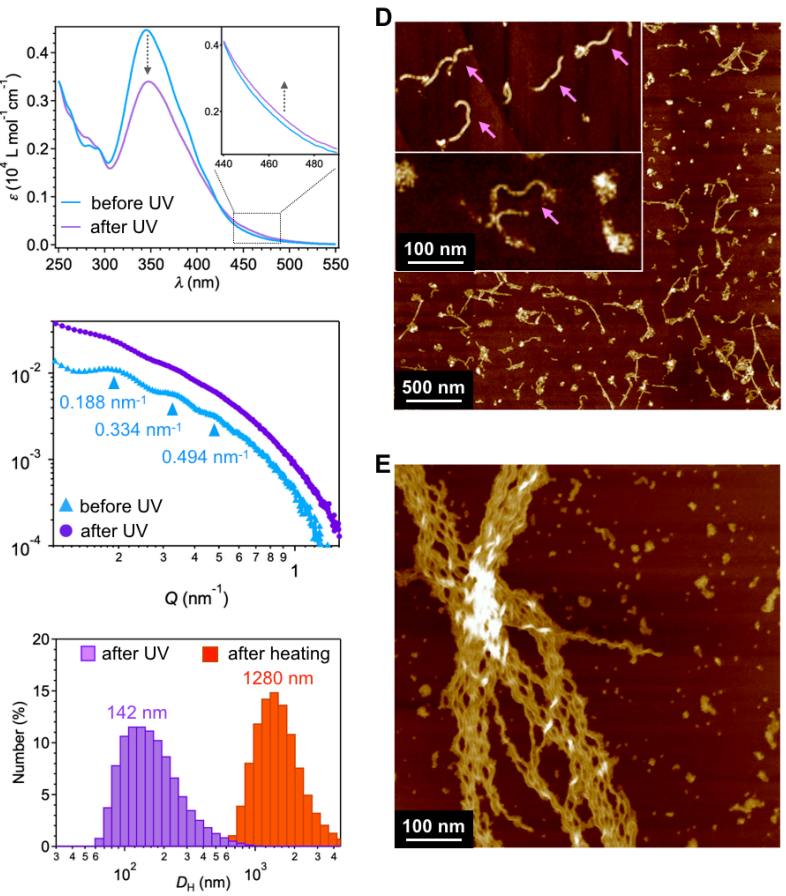

Figure. 6. (A) UV-Vis spectral change upon irradiation of the mixture of $\mathbf{S P}_{\text {random }}$ and $\mathbf{S} \mathbf{P}_{\text {ring }}\left(c_{\mathrm{T}}=1.0 \times 10^{-4} \mathrm{M}\right)$ with UV-light (from blue to purple spectra). The maximum absorption intensity (at $345 \mathrm{~nm}$ ) shows 12\% decrease after the UV irradiation, which corresponds to $17 \%$ trans-to-cis isomerization according to the NMR analysis (Figure S10). (B) Change of SAXS profiles upon irradiation of the mixture of $\mathbf{S P}_{\text {random }}$ and $\mathbf{S} \mathbf{P}_{\text {ring }}\left(c_{\mathrm{T}}=1.0 \times 10^{-4}\right.$ M) with UV-light (from blue to purple profiles). (C) DLS data of the UV-irradiated mixture of $\mathbf{S P}_{\text {random }}$ and $\mathbf{S P}_{\text {ring }}$ before (purple) and after heating (red) to $90{ }^{\circ} \mathrm{C}$. (D, E) AFM images of the UVirradiated mixture of $\mathbf{S P}_{\text {random }}$ and $\mathbf{S} \mathbf{P}_{\text {ring }}$ before (D) and after heating (E) to $90^{\circ} \mathrm{C}$.

\section{GONGLUSIONS}

In conclusion, we have demonstrated a topological impact on kinetic stability of metastable supramolecular polymers that can be thermally converted to extended fibers via monomeric state. We showed that the openended supramolecular polymers can be selectively transformed into insoluble crystalline fibers, while the circular counterpart remains intact. Since the longer circular species with obvious defect are also inert to thermal treatment, we attribute this topological impact on the kinetic stability of supramolecular polymers to the absence/presence of termini, not to the absence/presence of defect. Accordingly, light-induced ring-opening of the circular supramolecular polymers enabled the entire conversion of metastable species into thermodynamic products. Distinct kinetic stabilities of metastable supramolecular polymers with closed and open-ended 1Dtopologies demonstrated herein may be a hint to design artificial supramolecular systems whose internal diversity and complexity emerge not only from monomer structures but also topological features of assemblies.

\section{ASSOGIATED GONTENT}

\section{Supporting Information}

The Supporting Information is available free of charge on the ACS Publications website.

Experimental details synthetic procedures, compound characterization data, DSG thermograms, PXRD data, UV-vis spectra, GI-WAXD, thermography (PDF)

\section{AUTHOR INFORMATION}

\section{Corresponding Author \\ yagai@faculty.chiba-u.jp}

\section{ORCID}

Shiki Yagai: 0000-0002-4786-8603

Notes

The authors declare no competing financial interest.

\section{ACKNOWLEDGMENT}

This work was supported by a Grant-in-Aid for Scientific Research on Innovative Areas " $\pi$-System Figuration: Control of Electron and Structural Dynamism for Innovative Functions" from Japan Society for the Promotion of Science (JSPS) JP26102010. SY thanks Konika Minolta Science and Technology Foundation for partial financial support. The synchrotron XRD experiments were performed at the BL45XU in the SPring-8 with the approval of the RIKEN SPring-8 Center (proposal number 20170055).

\section{REFERENGES}

(1) (a) Korevaar, P. A.; George, S. J.; Markvoort, A. J.; Smulders, M. M. J.; Hilbers, P. A. J.; Schenning, A. P. H. J.; De Greef, T. F. A.; Meijer, E. W. Pathway complexity in supramolecular polymerization. Nature 2012, 481, 492-496. (b) De Greef, T. F. A.; Smulders, M. M. J.; Wolffs, M.; Schenning, A. P. H. J.; Sijbesma, R. P.; Meijer, E. W. Supramolecular Polymerization. Chem. Rev. 2009, 109, 5687-5754. (c) Sorrenti, A.; Leira-Iglesias, J.; Markvoort, A. J.; de Greef, T. F. A.; Hermans, T. M. Nonequilibrium supramolecular polymerization. Chem. Soc. Rev. 2017, $46,5476-5490$

(2) (a) Kang, J.; Miyajima, D.; Mori, T.; Inoue, Y.; Itoh, Y.; Aida, T. A rational strategy for the realization of chain-growth supramolecular polymerization. Science 2015, 347, 646-651. (b) Ogi, S.; Stepanenko, V.; Sugiyasu, K.; Takeuchi, M.; Würthner, F. Mechanism of Self-Assembly Process and Seeded Supramolecular Polymerization of Perylene Bisimide Organogelator. 7. Am. Chem. Soc. 2015, 137, 3300-3307. (c) Ogi, S.; Stepanenko, V.; Thein, J.; Würthner, F. Impact of Alkyl Spacer Length on Aggregation Pathways in Kinetically Controlled Supramolecular 
Polymerization. 7. Am. Chem. Soc. 2016, 138, 670-678. (d) Greciano, E. E.; Matarranz, B.; Sánchez, L. Pathway Complexity Versus Hierarchical Self-Assembly in $\mathcal{N}$-Annulated Perylenes: Structural Effects in Seeded Supramolecular Polymerization. $A n^{-}$ gew. Chem., Int. Ed. 2018, 57, 4697-4701.

(3) (a) Ogi, S.; Sugiyasu, K.; Manna, S.; Samitsu, S.; Takeuchi, M. Living supramolecular polymerization realized through a biomimetic approach. Nat. Chem. 2014, 6, 188-195. (b) Krieg, E.; Weissman, H.; Shimoni, E.; Bar On (Ustinov), A.; Rybtchinski, B. Understanding the Effect of Fluorocarbons in Aqueous Supramolecular Polymerization: Ultrastrong Noncovalent Binding and Cooperativity. F. Am. Chem. Soc. 2014, 136, 9443-9452.

(4) (a) Yamamoto, T.; Tezuka, Y. Topological polymer chemistry: a cyclic approach toward novel polymer properties and functions. Polym. Chem. 2011, 2, 1930-1941. (b) Peurifoy, S. R.; Guzman, C. X.; Braunschweig, A. B. Topology, assembly, and electronics: three pillars for designing supramolecular polymers with emergent optoelectronic behavior. Polym. Chem. 2015, 6, 5529-5539.

(5) Yagai, S.; Kitamoto, Y.; Datta, S.; Adhikari, B. Supramolecular Polymers Capable of Controlling Their Topology. Acc. Chem. Res. 2019, DOI: 10.1021 /acs.accounts.8b00660.

(6) (a) Yagai, S.; Goto, Y.; Lin, X.; Karatsu, T.; Kitamura, A.; Kuzuhara, D.; Yamada, H.; Kikkawa, Y.; Saeki, A.; Seki, S. Self-Organization of Hydrogen-Bonding Naphthalene Chromophores into J-type Nanorings and H-type Nanorods: Impact of Regioisomerism. Angew. Chem., Int. Ed. 2012, 51, 6643-6647. (b) Hollamby, M. J.; Aratsu, K.; Pauw, B. R.; Rogers, S. E.; Smith, A. J.; Yamauchi, M.; Lin, X.; Yagai, S. Simultaneous SAXS and SANS Analysis for the Detection of Toroidal Supramolecular Polymers Composed of Noncovalent Supermacrocycles in Solution. Angew. Chem., Int. Ed. 2016, 55, 9890-9893. (c) Aratsu, K.; Yagai, S. Self-Sorting of Rosette-Forming Naphthalene Barbiturates into Distinct Toroidal Assemblies. ChemPlusChem 2019, 84, DOI: 10.1002/cplu.201900044.

(7) Adhikari, B.; Yamada, Y.; Yamauchi, M.; Wakita, K.; Lin, X.; Aratsu, K.; Ohba, T.; Karatsu, T.; Hollamby, M. J.; Shimizu, N.; Takagi, H.; Haruki, R.; Adachi, S.-i.; Yagai, S. Lightinduced unfolding and refolding of supramolecular polymer nanofibres. Nat. Commun. 2017, 8, 15254.

(8) Prabhu, D. D.; Aratsu, K.; Kitamoto, Y.; Ouchi, H.; Ohba, T.; Hollamby, M. J.; Shimizu, N.; Takagi, H.; Haruki, R.; Adachi, S.-i.; Yagai, S. Self-folding of supramolecular polymers into bioinspired topology. Sci. Adv. 2018, 4, No. eaat8466.

(9) (a) Jonkheijm, P.; van der Schoot, P.; Schenning, A. P. H. J.; Meijer, E. W. Probing the Solvent-Assisted Nucleation Pathway in Chemical Self-Assembly. Science 2006, 313, 80-83. (b)
Smulders, M. M. J.; Nieuwenhuizen, M. M. L.; de Greef, T. F. A.; van der Schoot, P.; Schenning, A. P. H. J.; Meijer, E. W. How to Distinguish Isodesmic from Cooperative Supramolecular Polymerisation. Chem. -Eur. F. 2010, 16, 362-367.

(10) Ogi, S.; Stepanenko, V.; Sugiyasu, K.; Takeuchi, M.; Würthner, F. Mechanism of Self-Assembly Process and Seeded Supramolecular Polymerization of Perylene Bisimide Organogelator. F. Am. Chem. Soc. 2015, 137, 3300-3307.

(11) MacDonald J. G.; Whitesides, G. M. Solid-State Structures of Hydrogen-Bonded Tapes Based on Cyclic Secondary Diamides. Chem. Rev. 1994, 94, 2383-2420.

(12) (a) Silly, F.; Aratsu, K.; Yagai, S. Two-Dimensional Chiral SelfAssembly of Barbituric-Acid-Functionalized Naphthelene Derivatives. F. Phys. Chem. C 2018, 122, 6412-6416. (b) Yagai, S.; Nakano, Y.; Seki, S.; Asano, A.; Okubo, T.; Isoshima, T.; Karatsu, T.; Kitamura, A.; Kikkawa, Y. Supramolecularly Engineered Aggregation of a Dipolar Dye: Vesicular and Ribbonlike Architectures. Angew. Chem., Int. Ed. 2010, 49, 9990-9994. (c) Lin, X.; Suzuki, M.; Gushiken, M.; Yamauchi, M.; Karatsu, T.; Kizaki, T.; Tani, Y.; Nakayama, K.-i.; Suzuki, M.; Yamada, H.; Kajitani, T.; Fukushima, T.; Kikkawa, Y.; Yagai, S. Highfidelity self-assembly pathways for hydrogen-bonding molecular semiconductors. Sci. Rep. 2017, 7, 43098. (d) Ouchi, H.; Kizaki, T.; Yamato, M.; Lin, X.; Hoshi, N.; Silly, F.; Kajitani, T.; Fukushima, T.; Nakayama, K.-i.; Yagai, S. Impact of helical organization on the photovoltaic properties of oligothiophene supramolecular polymers. Chem. Sci. 2018, 9, 3638-3643.

(13) Albertazzi, L.; van der Zwaag, D.; Leenders, C. M. A.; Fitzner, R.; van der Hofstad, R. W.; Meijer, E. W. Probing Exchange Pathways in One-Dimensional Aggregates with SuperResolution Microscopy. Science 2014, 344, 491-495.

(14) Bochicchio, D.; Salvalaglio, M.; Pavan, G. M. Into the Dynamics of a Supramolecular Polymer at Submolecular Resolution. Nat. Commun. 2017, 8, 147.

(15) Jung, S. H.; Bochicchio, D.; Pavan, G. M.; Takeuchi, M.; Sugiyasu, K. A Block Supramolecular Polymer and Its Kinetically Enhanced Stability. F. Am. Chem. Soc. 2018, 140, 10570-10577.

(16) Adhikari, B.; Aratsu, K.; Davis, J.; Yagai, S. Photoresponsive Circular Supramolecular Polymers: A Topological Trap and Photoinduced Ring-Opening Elongation. Angerw. Chem., Int. Ed. 2019, 58, 3764-3768. 
Authors are required to submit a graphic entry for the Table of Contents (TOC) that, in conjunction with the manuscript title, should give the reader a representative idea of one of the following: A key structure, reaction, equation, concept, or theorem, etc., that is discussed in the manuscript. Consult the journal's Instructions for Authors for TOC graphic specifications.

Insert Table of Contents artwork here 